\title{
High Compliance With Surgical Site Infection (SSI) Prevention Bundle Reduces Incisional SSI After Colorectal Surgery
}

\author{
Varut Lohsiriwat \\ Colorectal Surgery Unit, Department of Surgery, Faculty of Medicine Siriraj Hospital, Mahidol University, Bangkok, Thailand
}

Purpose: This study aimed to evaluate association between compliance with surgical site infection (SSI) prevention bundle and the development of superficial or deep incisional SSI following colorectal surgery and to evaluate the impact of incisional SSI on surgical outcomes.

Methods: A prospectively collected database of consecutive patients undergoing elective colectomy and/or proctectomy from 2011 to 2019 in a university hospital was reviewed. The association between compliance with Thailand's SSI Prevention Bundle (10 level-1A interventions) and the incidence of incisional SSI was determined. Surgical outcomes were compared between those with incisional SSI and those without.

Results: This study included 600 patients with a median age of 64 years (range, 18-102 years). Some 126 patients (21.0\%) had stoma formation and $52(8.7 \%)$ underwent laparoscopy. The incidence of incisional SSI was $5.5 \%(\mathrm{n}=33 ; 32$ superficial incisional SSI and 1 deep incisional SSI). Higher compliance with care bundle tended to decrease incisional SSI $(\mathrm{P}=0.20)$. In multivariate analysis, compliance of $70 \%$ or more was the only dependent factor for reducing incisional SSI (odds ratio, $0.39 ; 95 \%$ confidence interval, 0.15 to $0.99 ; \mathrm{P}=0.047$ ). None of individual interventions were significantly associated with a lower probability of incisional SSI. Compared with counterparts, patients with incisional SSI had a 2-day longer length of postoperative stay ( 6 day vs. 4 day, $\mathrm{P}<0.001$ ) but comparable time for gastrointestinal recovery and similar rate of 30-day mortality or readmission.

Conclusion: High compliance with SSI prevention bundle (especially $\geq 70 \%$ ) reduced incisional SSI after colorectal surgery.

\section{Keywords: Compliance; Surgical wound infection; Colon; Rectum; Surgery}

\section{INTRODUCTION}

Surgical site infection (SSI) is the most common healthcare-associated infection accounting for $30 \%$ of overall nosocomial infections [1]. Among various intraabdominal operations, colorectal surgery had the highest rate of SSI [2]. It is evidence that patients developing SSI after colorectal surgery had a significantly longer length of hospital stay than those without (i.e., 8 days longer for

Received: Dec 30, 2019 - Revised: Mar 27, 2020 • Accepted: Apr 10, 2020 Correspondence to: Varut Lohsiriwat, M.D.

Department of Surgery, Faculty of Medicine Siriraj Hospital, Mahidol University, 2 Wang Luang Road, Bangkok 10700, Thailand

Tel: +66-2-4198005, Fax: +66-2-4121370

E-mail: bolloon@hotmail.com

ORCID: https://orcid.org/0000-0002-2252-9509

(C) 2021 The Korean Society of Coloproctology

This is an open-access article distributed under the terms of the Creative Commons Attribution NonCommercial License (https://creativecommons.org/licenses/by-nc/4.0) which permits unrestricted noncommercial use, distribution, and reproduction in any medium, provided the original work is properly cited. incisional SSI [3] and 24 days longer for organ/space SSI [4]). Moreover, patients with SSI were found to have 0.5 to 0.6 disability-adjusted life year [1]. In the recent years, there are several new or revised guidelines for the prevention of SSI published by many organizations such as the World Health Organization (WHO) [5, 6], the Centers for Disease Control and Prevention (CDC) [7], the United Kingdom's National Institute for Health and Care Excellence [8], and the Asia Pacific Society of Infection Control [9]. However, some have criticized these guidelines as they recommend too many interventions which are difficult to police and monitor, and some manoeuvers are irrelevant to some countries.

As a result, many local authorities have a policy to use their own care bundles (which usually include some key interventions from these guidelines) and show a significant reduction in the incidence of SSI after the implementation of these care bundles [10, 11]. In early 2020, the Surgical Infection Society of Thailand, in association with many Thai health organizations including the Society of Colorectal Surgeons of Thailand, has published 'Thai- 
land's SSI Prevention Bundle' which included 10 recommended interventions-mostly based on level 1A evidence [12]. This care bundle focuses on the prevention of 'incisional' SSI which remains prevalent in Thailand, particularly that following colorectal surgery [3]. Strategies for the prevention of organ/space SSI (e.g., anastomotic leakage and intraabdominal collection) are not covered by this care bundle because the development of organ/space SSI mainly depends on not only patient and disease factors but also surgical techniques $[4,13]$.

Briefly, 'Thailand's SSI Prevention Bundle' includes preoperative smoking cessation, weight-based antibiotic prophylaxis with proper timing and redosing of antibiotics, appropriate hair removal, skin preparation with alcohol-based solution, wound protectors for abdominal surgery, antimicrobial-coated sutures for abdominal wall closure, perioperative glucose control, maintenance of intraoperative normothermia, perioperative maintenance of oxygen saturation at least $92 \%$, and changing contaminated gloves and surgical instruments before wound closure [12].

It is well-known that guideline recommendations based on level 1A evidence are important but the cumulative effect of several such interventions in care bundles has hardly been evaluated. Therefore, this study primarily aimed to evaluate the impact of compliance with care bundles (Thailand's SSI Prevention Bundle) on the incidence of incisional SSI following major colorectal operations. The secondary objective of this study was to determine which individual interventions had a significant or greater SSI risk reduction and to evaluate the impact of incisional SSI on short-term surgical outcomes.

\section{METHODS}

\section{Patients}

Patient's data were extracted from a single surgeon's (the author) prospectively collected database registry of consecutive patients undergoing elective major colorectal operations (colectomy and/ or proctectomy) within an enhanced recovery after surgery (ERAS) program from January 2011 to November 2019 in a tertiary university hospital in Bangkok, Thailand. Patients with complicated intraabdominal infection and those without primary wound closure were excluded. The study was approved by the Institutional Ethics Committee of Faculty of Medicine Siriraj Hospital (Si482/2560) and written informed consent was obtained from each patient.

\section{Surgery and ERAS protocol}

All patients were operated on and treated by a board-certified colorectal surgeon and his team with an established ERAS protocol which was previously described in detail elsewhere $[14,15]$. Of note, a hospital-based care bundle for the prevention of incisional SSI is a part of full ERAS protocol and practically comparable to Thailand's SSI Prevention Bundle [12]. Moreover, the WHO surgical safety checklist has been introduced in our theater since late 2010. Regarding surgical techniques, extracorporeal anastomosis was performed for laparoscopic colectomy and intracorporeal anastomosis was done for laparoscopic rectal operations. Either stapled or hand-sewn anastomosis was performed for open colorectal operations. All incisions were closed layer by layer with appropriate suture materials except the use of staplers for skin approximation. It is worth noting that the small bites technique was used for abdominal fascial closure without the use of a prophylactic mesh or negative pressure wound therapy.

\section{Data collection}

Data collection included patients' characteristics, operative details, and postoperative outcomes. Patients' characteristics involved age, sex, body mass index, American Society of Anesthesiologists (ASA) physical status classification, indication for surgery, and CR-POSSUM score (ColoRectal Physiological and Operative Severity Score for the enUmeration of Mortality and Morbidity). Operative details included type of operation, operative time, and estimated blood loss. Overall compliance with Thailand's SSI Prevention Bundle of each patient was determined based on the published care bundle in 2020 [12]. Postoperative outcomes included incisional SSI which was diagnosed using the CDC criteria [16], postoperative complications (graded I to $\mathrm{V}$ according to the Clavien-Dindo classification system) [17], time to first defecation, time to resume solid diet, length of postoperative stay, and death and readmission within 30 days after an operation.

\section{Outcome measures}

The primary objective of the study was to evaluate the impact of compliance with Thailand's SSI Prevention Bundle on the incidence of incisional SSI following major colorectal operations within an ERAS protocol. The secondary objective of this study was to determine which individual interventions had a significant SSI risk reduction and the impact of incisional SSI on short-term surgical outcomes.

\section{Statistical analysis}

All statistical analyses were performed using the PASW Statistics software (ver. 18.0 for Windows, IBM Corp., Armonk, NY, USA). Continuous variables were expressed as mean \pm standard deviation or median (range or interquartile range [IQR]), and were compared using the Student t-test or Mann-Whitney U-test. Oneway analysis of variance was used to compare means among groups. Categorical data were expressed as number (percentage) and were compared using the Pearson chi-square test or Fisher exact probability test. Liner-by-linear association was used to test for trends of compliance with the care bundle. The univariate relation between each independent variable and incisional SSI was tested using binary logistic regression and the odds ratio (OR) with 95\% confidence interval (CI) for each variable was given. Potential variables $(\mathrm{P}<0.20)$ in the univariate analysis were included in a multivariate model of logistic regression [18]. A P- 
value of $<0.05$ was considered statistically significant.

\section{RESULTS}

\section{Patients' characteristics and operative details}

This study included 600 patients with average age of 63 years (range, 18 to 102 years) (Fig. 1). Of these patients, 324 (54.0\%) were male and $121(20.2 \%)$ had ASA classification of $\geq$ III. Some patients $(555,92.5 \%)$ underwent surgery for colorectal malignancy and 126 patients $(21.0 \%)$ had either temporary or permanent stoma formation. Eighty-two patients (13.7\%) had multivisceral organ resection. Fifty-two patients $(8.7 \%)$ underwent laparoscopy. Median operative time was 180 minutes (IQR, 150 to 240 minutes). Median blood loss was $150 \mathrm{~mL}$ (range, 50 to $300 \mathrm{~mL}$ ). Patients' characteristics and operative details were comparable between those with incisional SSI and those without except longer operative time in patients with incisional SSI (Table 1). Potential patient-related and treatment-related variables associated with incisional SSI were analyzed by univariate analysis and shown in Table 2.

\section{Overall outcomes}

Overall postoperative complication and severe complication were noted in $16.7 \%(n=100)$ and $3.7 \%(n=22)$, respectively. Rate of incisional SSI was 5.5\% $(\mathrm{n}=33 ; 32$ superficial incisional SSI and 1 deep incisional SSI). Rate of organ/space SSI was $2.2 \%(n=13 ; 7$ anastomotic leakages and 6 intraabdominal collections). Three patients died (1 perioperative acute myocardial infection, 1 sepsis, and 1 multiorgan failure) accounting for a 30-day mortality of $0.5 \%$. Median length of postoperative stay was 4 days (IQR, 3 to 5 days). Fifteen patients (2.5\%) had unplanned 30-day readmission.

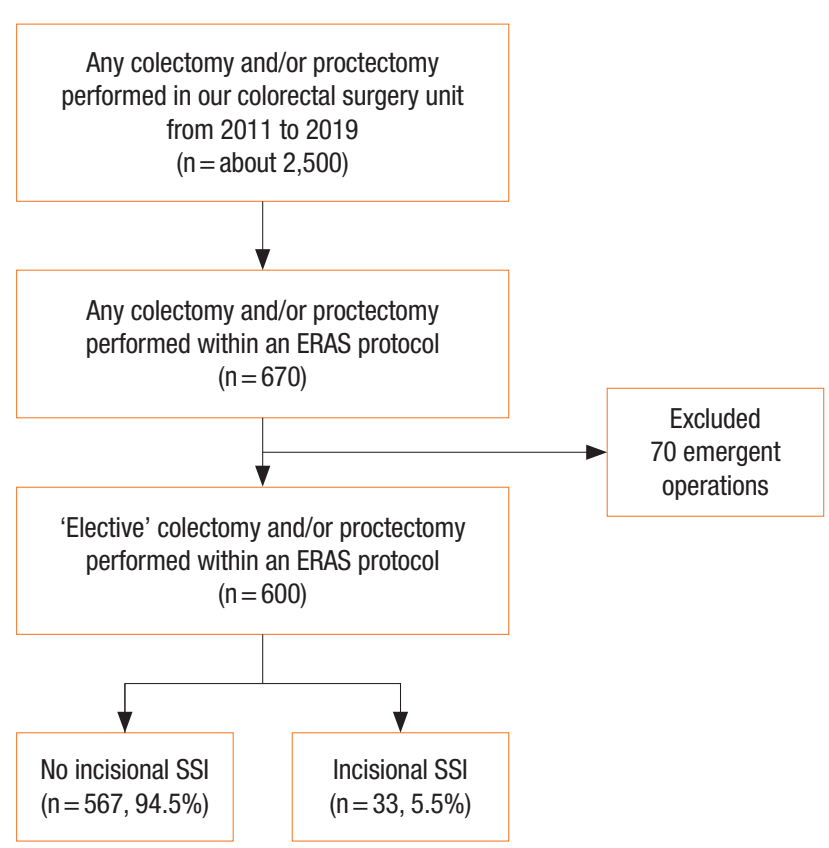

Fig. 1. Flow diagram of patients included in this study. ERAS, enhanced recovery after surgery; SSI, surgical site infection.

Table 1. Patient characteristics and operative details

\begin{tabular}{|c|c|c|c|c|}
\hline Variable & All $(n=600)$ & No incisional SSI $(n=567)$ & Incisional SSI $(n=33)$ & P-value \\
\hline Age (yr) & $63.3 \pm 13.0$ & $63.4 \pm 13.0$ & $61.7 \pm 14.2$ & 0.487 \\
\hline Male sex & $324(54.0)$ & $306(54.0)$ & $18(54.5)$ & 0.948 \\
\hline Body mass index $\left(\mathrm{kg} / \mathrm{m}^{2}\right)$ & $22.9 \pm 3.9$ & $22.9 \pm 3.9$ & $23.4 \pm 4.5$ & 0.527 \\
\hline ASA PS classification, $\geq \mid$ III & 121 (20.2) & $111(19.6)$ & $10(30.3)$ & 0.135 \\
\hline CR-POSSUM predicting mortality & $1.9(1.3-2.7)$ & $1.9(1.3-2.7)$ & $1.7(1.3-3.4)$ & 0.730 \\
\hline Preoperative hematocrit (\%) & $36.6 \pm 5.3$ & $36.6 \pm 5.3$ & $37.1 \pm 5.3$ & 0.570 \\
\hline Preoperative serum albumin (g/dL) & $4.0 \pm 1.6$ & $4.0 \pm 1.7$ & $3.6 \pm 0.6$ & 0.173 \\
\hline Colorectal cancer surgery & $555(92.5)$ & $523(92.2)$ & $32(97.0)$ & 0.501 \\
\hline Rectal surgery & $321(53.5)$ & $304(53.6)$ & $17(51.5)$ & 0.814 \\
\hline Surgery with stoma formation & $126(21.0)$ & $118(20.8)$ & $8(24.2)$ & 0.638 \\
\hline Multivisceral organ resection & $82(13.7)$ & $74(13.1)$ & $8(24.2)$ & 0.111 \\
\hline Laparoscopy & $52(8.7)$ & $52(9.2)$ & $0(0)$ & 0.102 \\
\hline Operative time (min) & $180(150-240)$ & $180(150-240)$ & $220(180-300)$ & $0.002^{\mathrm{a}}$ \\
\hline Estimated blood loss (mL) & $150(50-300)$ & $150(50-300)$ & $200(90-400)$ & 0.061 \\
\hline
\end{tabular}

Values are presented as mean \pm standard deviation, number (\%), or median (interquartile range).

SSI, surgical site infection; ASA, American Society of Anesthesiologists; PS, physical status; CR-POSSUM, ColoRectal Physiological and Operative Severity Score for the enUmeration of Mortality and Morbidity.

ap $<0.05$. 


\section{Association of compliance and clinical outcomes}

Overall median compliance with Thailand's SSI Prevention Bundle was $80 \%$ (range, $50.0 \%$ to $100 \%$ ). Higher compliance had a nonsignificant trend for reducing the incidence of incisional SSI $(\mathrm{P}=0.20)$ (Fig. 2). In multivariate analysis, compliance of $70 \%$ or more was the only dependent factor for reducing incisional SSI with an odd ratio of 0.39 ( $95 \% \mathrm{CI}, 0.15$ to $0.99 ; \mathrm{P}=0.047$ ) (Table 2). The highest compliance of individual bundle component was preoperative smoking cessation (100\%), alcohol-based antiseptic solution for skin preparation (100\%), and appropriate removal of

Table 2. Potential patient-related and treatment-related variables associated with incisional surgical site infection (SSI)

\begin{tabular}{|c|c|c|c|c|c|c|}
\hline \multirow{2}{*}{ Variable } & \multirow{2}{*}{ No. of patients (\%) } & \multirow{2}{*}{ No. of SSI (\%) } & \multicolumn{2}{|c|}{ Univariate analysis } & \multicolumn{2}{|c|}{ Multivariate analysis } \\
\hline & & & OR $(95 \% \mathrm{Cl})$ & P-value & OR (95\% Cl) & P-value \\
\hline Compliance with protocol & & & & 0.040 & & 0.047 \\
\hline$<70 \%$ & $51(8.5)$ & $6(11.8)$ & 1.00 & & 1.00 & \\
\hline$\geq 70 \%$ & $549(91.5)$ & $27(4.9)$ & $0.39(0.15-0.99)$ & & $0.38(0.15-0.99)$ & \\
\hline ASA PS classification & & & & 0.135 & & NS \\
\hline | and || & $479(79.8)$ & $23(4.8)$ & 1.00 & & & \\
\hline$\geq|I|$ & $121(20.2)$ & $10(8.3)$ & $1.79(0.83-3.86)$ & & & \\
\hline Laparoscopic surgery & & & & 0.102 & & NS \\
\hline No & $548(91.3)$ & $33(6.0)$ & 1.00 & & & \\
\hline Yes & $52(8.7)$ & $0(0)$ & $N A^{a}$ & & & \\
\hline Multivisceral organ resection & & & & 0.111 & & NS \\
\hline No & $518(86.3)$ & $25(4.8)$ & 1.00 & & & \\
\hline Yes & $82(13.7)$ & $8(9.8)$ & $2.13(0.93-4.90)$ & & & \\
\hline Operative time (hr) & & & & 0.100 & & NS \\
\hline$\leq 4$ & 469 (78.2) & $22(4.7)$ & 1.00 & & & \\
\hline$>4$ & $131(21.8)$ & $11(8.4)$ & $1.86(0.88-3.95)$ & & & \\
\hline Blood loss (mL) & & & & 0.110 & & NS \\
\hline$<300$ & $436(72.7)$ & $20(4.6)$ & 1.00 & & & \\
\hline$\geq 300$ & $164(27.3)$ & $13(7.9)$ & $1.79(0.87-3.69)$ & & & \\
\hline
\end{tabular}

Variables with a P-value of $<0.20$ were considered significant in univariate analysis and therefore defined as potential variables in this analysis. $\mathrm{OR}$, odds ratio; $\mathrm{Cl}$, confidence interval; ASA, American Society of Anesthesiologists; PS, physical status; NA, not available, NS, not significant.

a Odds ratio cannot be determine due to the zero event.

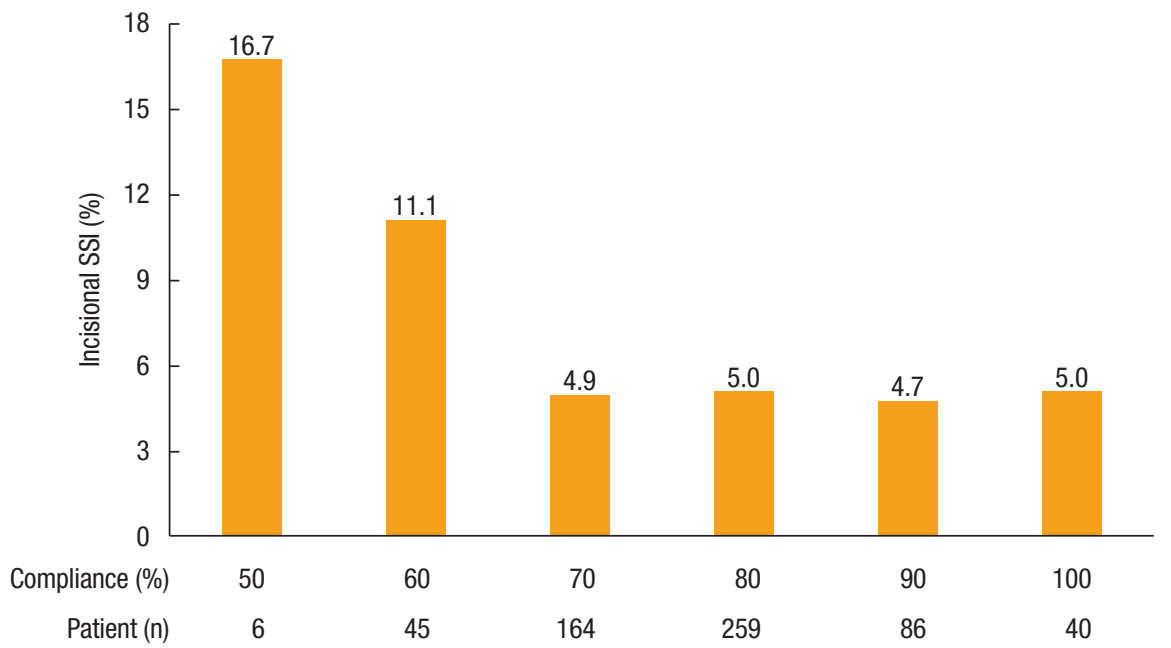

Fig. 2. Association between compliance with Thailand's Surgical Site Infection (SSI) Prevention Bundle and the incidence of incisional SSI (P=0.20). 


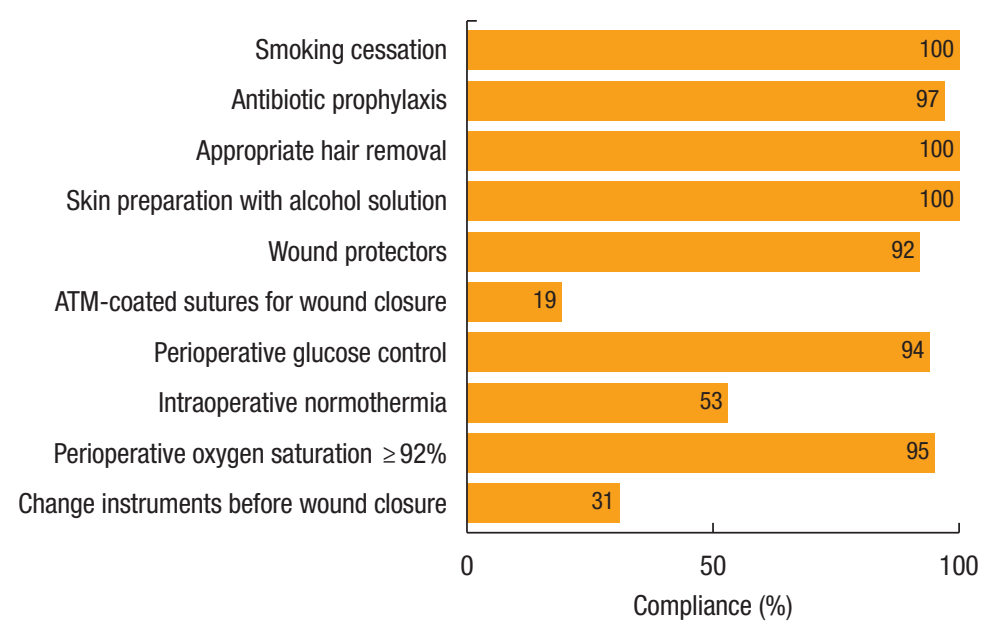

Fig. 3. Compliance with the individual interventions in Thailand's Surgical Site Infection Prevention Bundle. ATM, antimicrobial.

Table 3. Impact of incisional surgical site infection (SSI) and surgical outcomes

\begin{tabular}{lcccc}
\hline Variable & $\begin{array}{c}\text { All } \\
(\mathrm{n}=600)\end{array}$ & $\begin{array}{c}\text { No incisional } \\
\text { SSI }\end{array}$ & $\begin{array}{c}\text { Incisional } \\
\text { SSI }\end{array}$ & P-value \\
& $4(3-5)$ & $4(3-5)$ & $6(4-8)$ & $<0.001^{\text {a }}$ \\
\hline Postoperative stay (day) & $2(1-3)$ & $2(1-3)$ & $1(1-4)$ & 0.839 \\
Time to resume solid food (day) & $2(2-3)$ & $3(2-3)$ & $3(2-4)$ & 0.289 \\
Time to first defecation (day) & $3(3.0)$ & 0.156 \\
30-Day mortality & $3(0.5)$ & $2(0.4)$ & $1(30)$ & \\
30-Day unplanned readmission & $15(2.5)$ & $14(2.5)$ & $1(3.0)$ & 0.576 \\
\hline
\end{tabular}

Values are presented as number (\%) or median (interquartile range).

${ }^{\mathrm{a} P}<0.05$.

hair with clipper (100\%), whereas the use of antimicrobial-coated sutures for wound closure had the lowest compliance (19.3\%). Compliance with each recommended intervention is shown in Fig. 3. Of note, we cannot identify any of 10 bundle interventions that had a significant SSI risk reduction than the others.

\section{Impact of incisional SSI and surgical outcomes}

Patients with incisional SSI had a significantly 2-day longer length of median postoperative stay ( 6 vs. 4 day; $\mathrm{P}<0.001$ ) but comparable time to resume solid food and time to first defecation. There was no difference in 30-day mortality or unplanned readmission between patients with incisional SSI and those without (Table 3).

\section{DISCUSSION}

In this large, single-center, prospective study of 600 consecutive patients undergoing elective major colorectal operation within a perioperative care bundle, we found that patients with high compliance with the SSI prevention bundle $(\geq 70 \%)$ had a significant reduction in the rate of incisional SSI than those with lower com- pliance. However, we cannot identify any of the individual interventions that were significantly associated with a lower probability of incisional SSI thus suggesting that the favorable outcomes of SSI prevention bundle is based on the concepts of 'the aggregation of marginal gains' rather than the effect of individual intervention [19]. The aggregation of marginal gains can lead to dramatic improvement in patient's outcomes which has proven in this series, in which their overall rate for incisional SSI was 5.5\%. Of note, the rates of incisional SSI in our hospital before the implementation of perioperative care bundle were $12 \%$ for colon surgery and $16 \%$ for rectal surgery [3]. Meanwhile, patients with incisional SSI had a 2-day longer length of hospitalization than those without.

Similar to our study, a population-based study including over 400,000 surgical patients in the United States indicated that adherence to guidelines resulted in a decreased rate of postoperative infection including incisional SSI [20]. On the other hand, noncompliance with guidelines has been recognized as a risk factor for the development of SSI [21]. Although several studies have demonstrated an improvement in incisional SSI after the implementation of SSI prevention bundles $[10,11]$, our study has suggested that compliance with prevention bundle at least $70 \%$ has even better outcomes. The importance of adherence to SSI prevention bundle is supported by the Dutch national SSI surveillance network which demonstrated in 62,486 surgical patients that there was a $13 \%$ SSI risk reduction for each point increase in compliance level ranging from 0 to 4 [22].

Based on the 10 recommended interventions of Thailand's SSI Prevention Bundle, we achieved a 100\% compliance with preoperative smoking cessation, appropriate hair removal, and skin preparation with alcohol-based solution. It is possible that the highest achievement is due to the fact that we included only elective surgery, where smoking cessation at least 2 weeks before surgery is mandatory for every patient and all of these 3 interventions are parts of our well-established ERAS protocol $[14,15]$. 
Meanwhile, compliance with other recommended interventions was relatively high except the maintenance of intraoperative normothermia (53.3\% compliance), changes of contaminated gloves and surgical instruments before wound closure (31.2\% compliance), and the use of antimicrobial-coated sutures for wound closure ( $19.3 \%$ compliance).

Since a majority of our studied population underwent open surgery, it is known that laparotomy had a strong association with intraoperative hypothermia which adversely affected surgical outcomes such as high incidence of SSI $[23,24]$ and prolonged gastrointestinal recovery [25]. Therefore, a more robust protocol for maintaining perioperative normothermia especially in laparotomy is needed including preoperative active warming and use of warm intravenous fluid. Regarding the changes of contaminated gloves and surgical instruments before wound closure, we have had a policy of a 'new' set of instruments for wound closure for 3 years although there is no strong evidence on its effectiveness [6]. Due to this late implementation, overall compliance with this recommended intervention is relatively low in this series. Last, the use of antimicrobial-coated sutures for wound closure had the lowest compliance because of the availability and cost of these suture materials. Nevertheless, several meta-analyses of digestive surgery and nondigestive surgery have suggested that antimicrobial-coated sutures reduce incisional SSI by approximately $30 \%$ $[26,27]$ and they are potentially cost-saving across various wound types [28].

We failed to identify the contribution of individual interventions to the overall outcome because of lack of power. In fact, it is difficult to assess the effect of each intervention on SSI risk reduction even using a national database [22]. Regarding the impact of incisional SSI, patients with incisional SSI had a significantly 2-day longer length of hospitalization than those without. These adverse outcomes were even worse when an ERAS program was not applied (i.e., 8-day longer hospitalization if incisional SSI presented) [3].

This study has 2 major strengths. First, the data were extracted from a prospectively collected database in a university hospital with a well-established ERAS program and SSI prevention bundle. Second, this study provided detailed data on compliance and clinical outcomes of SSI prevention bundle in patients undergoing major colorectal surgery where incisional SSI remains prevalent $[2,3]$. However, some limitations of this study needed to be addressed. First, we do not examine the association of compliance with SSI prevention bundle and the development of organ/space for some reasons: (1) the fundamental strategy of this care bundle primarily focused on the prevention of incisional SSI; (2) the development of organ/space SSI mainly depends on patient and disease factors and surgical techniques [2,3], which may be difficulty to change or manipulate by perioperative care bundle; and (3) a larger number of studied population are required to achieve an adequate power of study on this subject because of the low incidence $(2.2 \%)$ of organ/space SSI in this series. Second, we do not examine whether mechanical bowel preparation with oral antibiotic affects the development of incisional SSI because there is no such practice in our institute although some recent studies have suggested that it reduces SSI rate by 30\% [29]. Third, we do not have clear explanation why there is no further reduction in the rate of incisional SSI in those with nearly $100 \%$ or $100 \%$ compliance with this care bundle. Perhaps, there are better strategies or interventions elsewhere to prevent SSI than the current care bundle. These findings could be a meaningful direction for future researches towards the achievement of 'zero' rate of SSI. Meanwhile, we need to balance between detailed or complex strategies for SSI prevention and pragmatic daily practices.

In conclusion, high compliance with SSI prevention bundle (especially $\geq 70 \%$ ) is crucial to reduce the development of incisional SSI. Since we cannot identify any of the individual interventions that were significantly associated with a lower probability of incisional SSI, these findings reflect that the combination of each individual intervention makes an effective SSI prevention bundle rather than the single intervention on its own.

\section{CONFLICT OF INTEREST}

No potential conflict of interest relevant to this article was reported.

\section{ACKNOWLEDGMENTS}

The author would like to thank Mr. Suthipol Udompunthurak from Clinical Epidemiology Unit the Office for Research and Development, Faculty of Medicine Siriraj Hospital, for his kind assistance with statistical analysis.

\section{REFERENCES}

1. Cassini A, Plachouras D, Eckmanns T, Abu Sin M, Blank HP, Ducomble $\mathrm{T}$, et al. Burden of six healthcare-associated infections on European population health: estimating incidence-based disability-adjusted life years through a population prevalence-based modelling study. PLoS Med 2016;13:e1002150.

2. Romy S, Eisenring MC, Bettschart V, Petignat C, Francioli P, Troillet N. Laparoscope use and surgical site infections in digestive surgery. Ann Surg 2008;247:627-32.

3. Lohsiriwat V, Lohsiriwat D. Antibiotic prophylaxis and incisional surgical site infection following colorectal cancer surgery: an analysis of 330 cases. J Med Assoc Thai 2009;92:12-6.

4. Lohsiriwat V, Lohsiriwat D, Boonnuch W, Chinswangwatanakul V, Akaraviputh T, Riansuwan W, et al. Outcomes of sphincter-saving operation for rectal cancer without protective stoma and pelvic drain, and risk factors for anastomotic leakage. Dig Surg 2008;25: 191-7.

5. Allegranzi B, Bischoff P, de Jonge S, Kubilay NZ, Zayed B, Gomes $\mathrm{SM}$, et al. New WHO recommendations on preoperative mea- 
sures for surgical site infection prevention: an evidence-based global perspective. Lancet Infect Dis 2016;16:e276-87.

6. Allegranzi B, Zayed B, Bischoff P, Kubilay NZ, de Jonge S, de Vries $F$, et al. New WHO recommendations on intraoperative and postoperative measures for surgical site infection prevention: an evidence-based global perspective. Lancet Infect Dis 2016;16:e288303.

7. Berríos-Torres SI, Umscheid CA, Bratzler DW, Leas B, Stone EC, Kelz RR, et al. Centers for Disease Control and Prevention Guideline for the Prevention of Surgical Site Infection, 2017. JAMA Surg 2017;152:784-91.

8. NICE Guideline Updates Team. Surgical site infections: prevention and treatment: NICE guideline (NG 125) [Internet]. London (UK): National Institute for Health and Care Excellence; 2019 [cited 2020 Aug 3]. Available from: https://www.nice.org.uk/guidance/ng125.

9. Ling ML, Apisarnthanarak A, Abbas A, Morikane K, Lee KY, Warrier A, et al. APSIC guidelines for the prevention of surgical site infections. Antimicrob Resist Infect Control 2019;8:174.

10. Zywot A, Lau CSM, Stephen Fletcher H, Paul S. Bundles prevent surgical site infections after colorectal surgery: meta-analysis and systematic review. J Gastrointest Surg 2017;21:1915-30.

11. Carter EB, Temming LA, Fowler S, Eppes C, Gross G, Srinivas $\mathrm{SK}$, et al. Evidence-based bundles and cesarean delivery surgical site infections: a systematic review and meta-analysis. Obstet Gynecol 2017;130:735-46.

12. Lohsiriwat V, Chinswangwatanakul V, Lohsiriwat $\mathrm{D}$, Rongrungruang Y, Malathum K, Ratanachai P, et al. Guidelines for the prevention of surgical site infection: the Surgical Infection Society of Thailand recommendations (executive summary). J Med Assoc Thai 2020;103:99-105.

13. Gomila A, Carratala J, Camprubi D, Shaw E, Badia JM, Cruz A, et al. Risk factors and outcomes of organ-space surgical site infections after elective colon and rectal surgery. Antimicrob Resist Infect Control 2017;6:40.

14. Lohsiriwat $\mathrm{V}$. The influence of preoperative nutritional status on the outcomes of an enhanced recovery after surgery (ERAS) programme for colorectal cancer surgery. Tech Coloproctol 2014;18: 1075-80.

15. Lohsiriwat V. Learning curve of enhanced recovery after surgery program in open colorectal surgery. World J Gastrointest Surg 2019;11:169-78.

16. Mangram AJ, Horan TC, Pearson ML, Silver LC, Jarvis WR. Guideline for prevention of surgical site infection, 1999. Hospital Infection Control Practices Advisory Committee. Infect Control Hosp Epidemiol 1999;20:250-78.

17. Dindo D, Demartines N, Clavien PA. Classification of surgical complications: a new proposal with evaluation in a cohort of 6336 patients and results of a survey. Ann Surg 2004;240:205-13.

18. Concato J, Feinstein AR, Holford TR. The risk of determining risk with multivariable models. Ann Intern Med 1993;118:20110.

19. Fleming IO, Garratt C, Guha R, Desai J, Chaubey S, Wang Y, et al. Aggregation of marginal gains in cardiac surgery: feasibility of a perioperative care bundle for enhanced recovery in cardiac surgical patients. J Cardiothorac Vasc Anesth 2016;30:665-70.

20. Stulberg JJ, Delaney CP, Neuhauser DV, Aron DC, Fu P, Koroukian SM. Adherence to surgical care improvement project measures and the association with postoperative infections. JAMA 2010; 303:2479-85.

21. Beldi G, Bisch-Knaden S, Banz V, Muhlemann K, Candinas D. Impact of intraoperative behavior on surgical site infections. Am J Surg 2009;198:157-62.

22. Koek MBG, Hopmans TEM, Soetens LC, Wille JC, Geerlings SE, Vos MC, et al. Adhering to a national surgical care bundle reduces the risk of surgical site infections. PLoS One 2017;12:e0184200.

23. Kurz A, Sessler DI, Lenhardt R. Perioperative normothermia to reduce the incidence of surgical-wound infection and shorten hospitalization. Study of Wound Infection and Temperature Group. N Engl J Med 1996;334:1209-15.

24. Seamon MJ, Wobb J, Gaughan JP, Kulp H, Kamel I, Dempsey DT. The effects of intraoperative hypothermia on surgical site infection: an analysis of 524 trauma laparotomies. Ann Surg 2012;255: 789-95.

25. Lohsiriwat V, Jaturanon P. Effect of intraoperative hypothermia on surgical outcomes after colorectal surgery within an enhanced recovery after surgery pathway. Siriraj Med J 2019;71:52-8.

26. De Jonge SW, Atema JJ, Solomkin JS, Boermeester MA. Metaanalysis and trial sequential analysis of triclosan-coated sutures for the prevention of surgical-site infection. Br J Surg 2017;104: e118-33.

27. Ahmed I, Boulton AJ, Rizvi S, Carlos W, Dickenson E, Smith NA, et al. The use of triclosan-coated sutures to prevent surgical site infections: a systematic review and meta-analysis of the literature. BMJ Open 2019;9:e029727.

28. Leaper DJ, Edmiston CE Jr, Holy CE. Meta-analysis of the potential economic impact following introduction of absorbable antimicrobial sutures. Br J Surg 2017;104:e134-44.

29. Toh JW, Phan K, Hitos K, Pathma-Nathan N, El-Khoury T, Richardson AJ, et al. Association of mechanical bowel preparation and oral antibiotics before elective colorectal surgery with surgical site infection: a network meta-analysis. JAMA Netw Open 2018;1: e183226. 